\title{
Liderazgo para el aprendizaje: ¿Qué tareas de los directores y directoras escolares son las que más inciden en el aprendizaje de los estudiantes?
}

Leadership for learning: What are the principals' tasks that most impact in the student learning?

\author{
Murillo, F. Javier ${ }^{(1)}$; Hernández-Castilla, Reyes
}

(1) Universidad Autónoma de Madrid.

\begin{abstract}
Resumen
El estudio sobre la distribución del tiempo de los directores escolares y sus efectos sobre el desempeño de los estudiantes es una productiva línea de investigación educativa que ha aportado datos interesantes para la mejora de los centros educativos. El presente estudio busca determinar la incidencia de la distribución del tiempo de los directivos escolares en el desempeño de sus estudiantes, e identificar los factores que inciden sobre dicha distribución. Para ello se hace una explotación especial de la Evaluación General Diagnóstica de Primaria realizada en España en 2009. Los resultados indican que los estudiantes de los centros cuyos directores dedican más tiempo a tareas pedagógicas obtienen significativamente mejores resultados que los que dedican a actividades administrativas. También que el género, edad y formación para el desempeño del directivo, así como la titularidad y el tamaño del centro son factores que inciden en la distribución de su tiempo.
\end{abstract}

Fecha de recepción 7 de Enero de 2015

Fecha de aprobación 15 de Marzo de 2015

Fecha de publicación 29 de Marzo de 2015

\section{Palabras clave:}

Dirección escolar, Distribución de tiempo, Organización escolar, Educación primaria

\footnotetext{
Abstract

The study of the time allocation of school principals and their effects on the performance of their students is a productive research line that has provided interesting educational information to school improvement. This study aims to determine the incidence of time allocation of school principals in the performance of their students, and to identify the factors that influence the distribution. For that purpose, special data mining of the General Diagnostic Primary Assessment held in Spain in 2009. The results show that students in schools whose principals spend more time on educational tasks get significantly better results than those who spend on administrative activities. Also gender, age and specific training for being principal, and the school ownership and size are factors than influence on time allocation.
}

Reception Date 2015 January 07

Approval Date 2015 March 15

Publication Date: 2015 March 29

\section{Keywords:}

School leadership, Task allocation, Management, Primary education

La distribución del tiempo de los directores y directoras de escuela en las diferentes tareas, y la forma en que esa distribución afecta a la mejora de la escuela y al desempeño de los estudiantes, es una fructífera línea de investigación educativa que se ha desarrollado

\section{Autor de contacto / Corresponding author}

Murillo, F. Javier. Facultad de Formación de Profesorado y Educación. Universidad Autónoma de Madrid. Avda. 
en todo el mundo desde hace más de 40 años (Lee y Hallinger, 2012). La hipótesis de fondo es que algunas tareas que desarrolla el directivo inciden más que otras en generar dinámicas en el centro educativo que hacen que éste consiga más y mejor sus objetivos. De esta forma la priorización de metas y objetivos constituye uno de los principales desafíos con los que tiene que enfrentarse un directivo en la actualidad (Goldring et al., 2008; Murillo, Barrio y Pérez-Albo, 1999; Silins y Mulford, 2010).

Sin embargo, tal y como han demostrado Lee y Hallinger (2012), tanto la distribución del tiempo de los directivos como su incidencia difieren de forma importante de un país a otro, por lo que, una vez más, no es posible conformarse con investigaciones hechas en otras realidades. El único estudio en España que analiza esa distribución y la relaciona con el desempeño de los estudiantes tiene ya 14 años de vida (Murillo y Barrio, 1999) así que conviene actualizarlo.

En esta investigación, usando datos recientes, oficiales y con una muestra representativa del Estado español, se busca profundizar en el conocimiento de la distribución del tiempo de los directivos españoles de centros de Educación Primaria y su incidencia en el aprendizaje de los estudiantes. Para ello se ha realizado una explotación especial de la base de datos de la Evaluación General de Diagnóstico de Educación Primaria desarrollada en España en 2009, utilizando Modelos Multinivel.

\section{Revisión de la literatura}

Aunque desde los años 60 es posible encontrar trabajos que abordan el estudio de la distribución del tiempo de los directores escolares, es a partir de la década de los 80 cuando se ha desarrollado una productiva línea de investigación educativa que busca profundizar tanto en su descripción, como en el análisis de sus efectos sobre la dinámica del centro. Entre los estudios más relevantes desarrollados en la década de los 80 se encuentran los de Burke (1980), Eberts y Stone (1988), Ghosey (1987), Hallet (1985),
Kmetz y Willower (1982), Martin y Willower (1981), y Osborne y Wiggins (1989). En la década de los 90 destacan las aportaciones de Gorman (1993) o Helps (1994); y, ya en este siglo, se encuentran trabajos como los de Buttram, Mead, Loftus, y Wilson (2008), Goldring at al. (2008), Horng, Klasik y Loeb (2010), Larry (2003), Murillo y Román (2013), Rayfield y Diamantes (2004), Robinson, Lloyd, y Rowe (2014); Spillane, Camburn y Pareja (2007), Taylor (2007), y Walker (2009).

En España destacan dos trabajos. Por un lado, el estudio de Murillo y Barrio (1999) con datos de 437 directores y directoras de Educación Primaria. Y, por otro, un reciente e interesante estudio desarrollado por LópezYáñez, García-Jiménez, Oliva-Rodríguez, Moreta-Jurado, y Bellerín (2014) sobre la actividad diaria de los directores y directoras de escuela.

Las metodologías usadas en estos trabajos son muy variadas. Entre ellas, se encuentra la observación, tanto estructurada como informal, de las actividades desarrolladas por el directivo (Martinko y Gardner, 1990), entrevistas en profundidad, estudios de encuesta (Eberts y Stone, 1988), investigaciones etnográficas (Wolcott, 1973), $\mathrm{y}$ diarios de auto-reporte (Goldring et al., 2008; López-Yañez et al. 2014; May, Huff, y Goldring, 2012). Pero destaca especialmente el uso de análisis secundarios de macroevaluaciones internacionales o nacionales tales como el Progress in International Reading Literacy Study (PIRLS) (Lee y Hallinger, 2012), el Trends in International Mathematics and Science Study (TIMMS) (Mullis, Martin y Foy, 2008), ambos de la IEA, el proyecto Indicators of Education Systems (INES) de la OCDE (Murillo y Barrio, 1999), y el Segundo Estudio Regional Comparativo y Explicativo (SERCE) de la UNESCO (Murillo y Román, 2013).

Esta línea se planteó como uno de sus primeros objetivos conocer qué porcentaje de tiempo dedican a cada tarea los directores y directoras de escuelas (Gordon, 1996; Walker, 2009). La idea que se desprende de él, 
independientemente del nivel o el país, es que se dedicaban prioritariamente a las tareas administrativas y burocráticas, y muy poco a las relaciones públicas y pedagógicas.

Para tener una idea de las cifras concretas, en Estados Unidos, Buttram, Mead, Loftus y Wilson (2008) encontraron que el 63,7\% del tiempo se destina a prácticas relacionadas con cuestiones de carácter administrativo; mientras que el $14 \%$ del tiempo lo dedican a actividades directamente ligadas a la instrucción (la planificación, el seguimiento a estudiantes y docentes, la participación en comités y en reuniones relacionadas con la docencia). También en el estudio intensivo sobre el papel de los líderes exitosos intermedios en Nueva Zelanda se realizó un análisis de las tareas realizadas por los mandos intermedios en la escuela y su posición dentro de ella (Marshall, 2014).

El TIMSS, tanto en su estudio de 2003 como en el del 2007, incluyó en sus cuestionarios de contexto una pregunta sobre la distribución del tiempo de los directores de los países participantes (Mullis, Martin, y Foy, 2008). Los resultados muestran, una vez más, la mayor dedicación a tareas administrativas frente a las puramente pedagógicas de los directivos (Tabla 1).

Tabla 1 - Promedio de la distribución del tiempo de los directivos participantes en el TIMSS 2007

\begin{tabular}{lcc}
\hline \multicolumn{1}{c}{ Tareas } & $\begin{array}{c}\mathbf{4}^{\mathbf{o}} \text { de } \\
\text { Primaria }\end{array}$ & $\begin{array}{c}\mathbf{8}^{\mathbf{o}} \mathbf{d e} \\
\text { Primaria }\end{array}$ \\
\hline Tareas Administrativas (Ej. Presupuesto, planificación, reuniones, contratación) & $32(, 2)$ & $30(, 2)$ \\
Liderazgo Pedagógico & $21(1,0)$ & $20(, 1)$ \\
Supervisión y evaluación de profesores y otro personal & $19(, 1)$ & $22(, 1)$ \\
Relaciones públicas y recaudación de fondos & $10(, 1)$ & $11(, 1)$ \\
Docencia & $11(, 1)$ & $9(, 1)$ \\
Otros & $7(, 1)$ & $8(, 1)$ \\
\hline
\end{tabular}

Fuente: Elaboración propia a partir de Mullis, Martin, y Foy (2008)

En América Latina sobresalen dos trabajos: por un lado, la Investigación Iberoamericana sobre Eficacia Escolar, que analizó el tiempo de los directivos de 91 escuelas de ocho países de la Región (Murillo, 2007). Éste encontró que la mayor parte del mismo lo emplean en tareas burocráticas (36,4\%), seguido de actividades de liderazgo pedagógico (20,1\%), el destinado a su desarrollo profesional es un $17,3 \%$, mientras que el dedicado a contactos con las familias corresponde al 16,1\%. Por otro, está el estudio de Murillo y Román (2013), con datos obtenidos de 2.580 directores y directoras de 16 países de la región. Sus resultados apuntan a que un $16 \%$ del tiempo lo dedican a tareas de supervisión y consejo de los profesores, un tiempo equivalente a prácticas de liderazgo pedagógico, un $25 \%$ a tareas administrativas y de gestión, tan solo un $12,2 \%$ a relaciones públicas, un 9,9\% en actividades de desarrollo personal $\mathrm{y}$, por último un $8,5 \%$ en conseguir recursos para la escuela.

En el contexto español, en los años 90 se realizaron varios estudios en este sentido (Antúnez, 1993; Armas, 1998; Gimeno, 1995; Murillo y Barrio, 1999). Los trabajos de Armas (1998) o de Gimeno (1995) usan como medida escalas de frecuencia de dedicación a determinadas tareas, y no cantidad de tiempo dedicado. Así, Gimeno (1995) encuentra que las actividades menos practicadas son de tipo pedagógico y de coordinación, mientras que las que presentan índices mayores de frecuencia son las tareas de gestión, difusión de información y control (Figura 1). 
Figura 1 - Estimación de las actividades realizadas por los directivos españoles



Fuente: Gimeno (1995: 199)

Quizá el estudio español más preciso hasta el momento sea el de Murillo y Barrio (1999) con datos de 437 directores de escuelas de Primaria de centros públicos, concertados y privados. En él se constata que el mayor porcentaje de tiempo se invierte en tareas relativas a la administración y la organización de su planificación (36,44\%), el 26,7\% a tareas de liderazgo pedagógico, un 15,6\% a la relación con las madres y padres de los estudiantes, algo menos al desarrollo personal $(14,95 \%)$ y la menor cantidad de tiempo, el $6,31 \%$, se destina a otras tareas (Figura 2 ).

Figura 2 - Distribución de las tareas no docentes de los directivos de centros de primaria en España

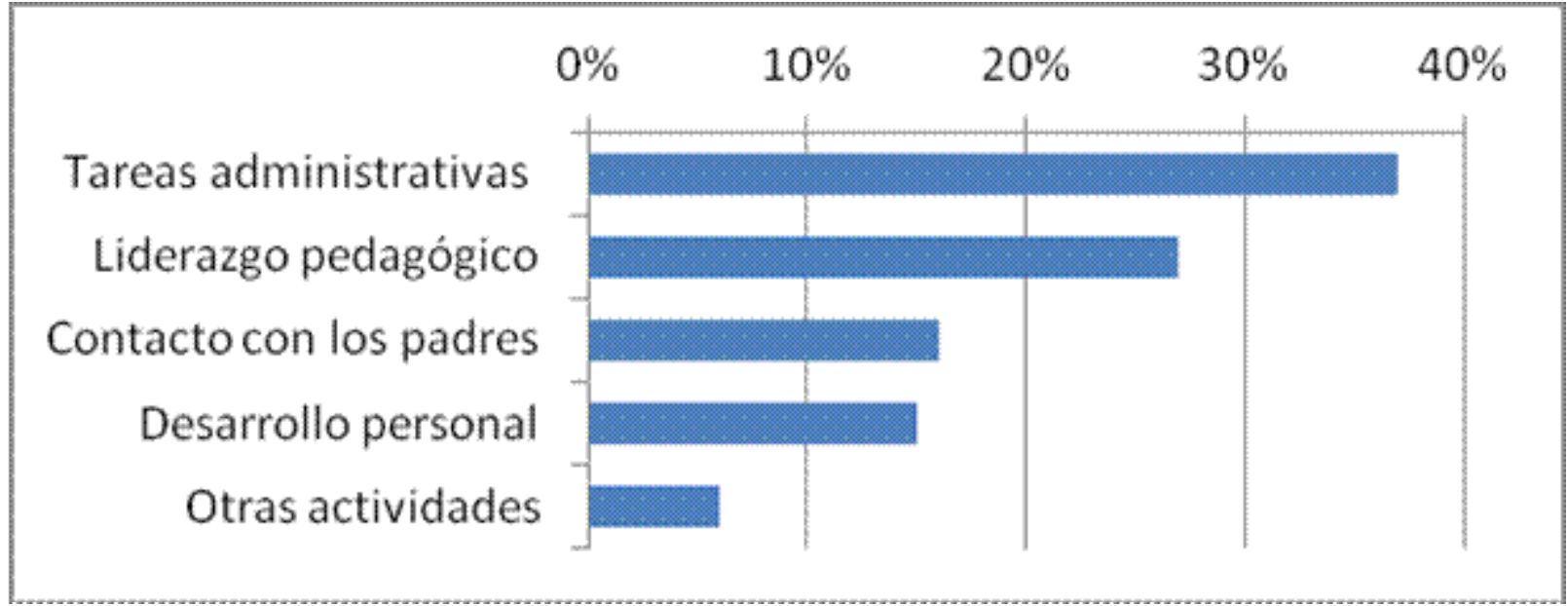

Fuente: Murillo y Barrio (1999:209)

Un segundo objetivo que se plantea esta línea de investigación es determinar qué factores están relacionados con la distribución del tiempo. Así se ha abordado la incidencia de variables tales como el género, la edad o la experiencia del directivo, el tamaño o la titularidad del centro.

En su tesis doctoral, la profesora Jaqueline Hallet (1985) se focalizó en determinar las diferencias en la distribución del tiempo de los 
directivos en función del su género. Y encontró que las mujeres dedican más tiempo a prácticas directamente ligadas al liderazgo pedagógico que los hombres y menos a las tareas administrativas. Resultados análogos a los encontrados anteriormente por Burke (1980) y Olsen (1980), y posteriormente por Wells (1993), y Murillo y Barrio (1999). Padilla (2008), en su estudio de 521 directoras de centros públicos andaluces, observó que la mayoría de las que acceden al puesto generalmente están libres de cargas familiares y son de mediana edad. Manifiestan que el tiempo dedicado a tareas de gestión sobre todo les afecta a su tiempo personal fuera del centro. Y, analizando las tareas de su liderazgo se determinó que es predominantemente pedagógico. Sin embargo, en el estudio realizado por Murillo y Román (2013), no encuentran diferencias significativas en función del género de los directivos.

Wells (1993), por su parte, incorporó otras variables además del género, entre ellas la experiencia en el cargo. Los datos son claros en determinar que los directivos más experimentados dedican más tiempo a cuestiones pedagógicas. Murillo y Barrio (1999) encontraron diferencias entre los directores de mayor edad, los cuales dedican más tiempo a las tareas administrativas y menos a la búsqueda de recursos para el centro. Resultados análogos a los hallados por Murillo y Román (2013).

En un reciente estudio, López-Yáñez et al. (2014) se analizó la distribución de las tareas desde una perspectiva de la colaboración entre la dirección y los miembros de la comunidad educativa, a través del registro reportado por los propios directores/as. Se ha observado que más de la mitad de las actividades (54\%) son realizadas con la participación de otros profesores del centro, estudiantes, la administración e incluso otros agentes de la comunidad educativa. Mientras que tan solo un $31 \%$ eran individuales. Como en otros estudios semejantes (Spillane, 2005) aparece que el patrón y la magnitud de la distribución del liderazgo guarda relación con el tipo de actividad de la que se trata. Concluyeron en este estudio que el coliderazgo está más presente en los centros de Educación Secundaria $(61,5 \%)$ que en los de Primaria $(49,4 \%)$, probablemente por la mayor autonomía del profesorado de Secundaria.

Otro factor que, según los diferentes estudios, parece tener relación con la distribución del tiempo de los directivos es el tamaño de la escuela. Y los resultados suelen apuntar a que cuanto más grande es la escuela, más tiempo dedican a tareas administrativas y menos a cuestiones pedagógicas (Burke, 1980; Wells, 1993). Sin embargo, Murillo y Barrio (1999) en su estudio en España, encontraron que los directivos con poca experiencia de centros públicos de tamaño medio dedican más tiempo a temas de carácter curricular. Por el contrario, los que menos se ocupan de tareas administrativas pertenecen a centros concertados pequeños y llevan poco tiempo desempeñando el puesto.

Mucho más interesante es conocer si esa distribución afecta al desempeño de los estudiantes. Horng, Klasik y Loeb (2010) encontraron que las escuelas de bajo rendimiento se diferencian en la distribución del tiempo de sus directores respecto a las de alto rendimiento. Así, descubrieron una relación significativa y positiva entre el tiempo dedicado a tareas de gestión de la organización tales como la contratación de personal y la distribución de recursos, y los resultados de los estudiantes. Desde otra perspectiva, Thorpe (2014) también ha explorado la división entre el liderazgo y la gestión educativa, y en este sentido cuales son las implicaciones que tienen en las escuelas, cuestionando la persistencia de la separación entre ambos conceptos.

La ya citada Investigación Iberoamericana sobre Eficacia Escolar (Murillo, 2007), halló que existe una relación estadísticamente significativa entre el tiempo que dedican los directivos a tareas relacionadas con el liderazgo pedagógico y un mayor desempeño de los estudiantes de esa escuela. 
Murillo y Román (2013), por último, con datos de 2.580 directivos de 16 países de América Latina, realizaron un análisis multinivel con tres niveles para el rendimiento en Matemáticas y Lectura. Allí se encontró que el porcentaje de tiempo que los directores $\mathrm{y}$ directoras emplean en el liderazgo pedagógico influye en el rendimiento de los estudiantes de Sexto Grado de Primaria en Matemáticas y en Lectura, así mismo en Lectura con los estudiantes de Tercer Grado, y mayor en Matemáticas que en Lectura.

Con esta investigación se pretende profundizar en el conocimiento de la distribución del tiempo de los directores y directoras de centros de Educación Primaria en España en las tareas de dirección, qué influye en esta distribución y qué incidencia tiene en el desempeño de los estudiantes. Formalmente busca alcanzar los siguientes objetivos:

- Determinar la incidencia de la distribución del tiempo de los directivos en el desempeño de los estudiantes de la escuela.

- Identificar los factores relativos a las características del directivo y del centro asociados a esa distribución.

\section{Metodología}

\section{Procedimiento}

Para alcanzar estos objetivos se realizó una explotación especial de los datos de la Evaluación de Diagnóstico General (EDG) de Educación Primaria realizada en 2009 por el actual Instituto Nacional de Evaluación Educativa, del Ministerio de Educación de España (Instituto de Evaluación, 2010). Junto con las pruebas de rendimiento en cuatro competencias básicas, se aplicó una serie de cuestionarios de contexto que aportaron información que no solo ayuda a contextualizar los resultados de desempeño obtenidos, sino que también aporta una información que permite profundizar en el conocimiento de las escuelas, los docentes y los estudiantes españoles de Educación Primaria.
Las variables utilizadas en el estudio fueron de varios tipos:

1. Distribución del tiempo de trabajo de los directivos: Porcentaje de tiempo dedicado a Tareas administrativas internas, a Tareas relacionadas con el currículo y la enseñanza, a Tareas relacionadas con las peticiones de las Administraciones, a Tareas relacionadas con reuniones con docentes o familias, y a otras Tareas.

2. Variables relativas a las características del directivo y del centro donde labora con el fin de determinar cómo influyen en la distribución de tiempo: Titularidad del centro (público/privado); Tamaño del centro (estimado a partir del número de docentes que trabajan en la escuela); ISEC de la escuela (promedio de la escuela del índice estadístico de estatus social, económico y cultural (ISEC), calculado a partir de las respuestas de los alumnos y de sus familias; expresado como un valor tipificado a partir de cuatro componentes: el nivel más alto de estudios de los padres, la profesión más alta de los padres, el número de libros en el domicilio familiar y el nivel de recursos domésticos; Género del directivo; Edad del directivo; Experiencia en funciones directivas; Formación específica sobre el cargo; y Satisfacción del director o directora con la escuela.

3. Variables del desempeño de los estudiantes, todas ellas estimadas mediante TRI y con una puntuación en una escala de media 500 puntos y desviación típica 100, en las siguientes áreas: Competencia en Comunicación Lingüística, Competencia Matemática, Competencia en el Conocimiento y la Interacción con el Mundo Físico, y Competencia Social y Ciudadana.

4. Variables de ajuste: ISEC de la familia del estudiante, ISEC de la escuela, titularidad del centro, Género del estudiante y Preescolarización del estudiante. 


\section{Muestra}

La muestra final utilizada para esta investigación estuvo conformada por 874 directores y directoras de escuelas de Educación Primaria y 28.708 estudiantes de $4^{\circ}$ curso de Educación Primaria. El muestreo fue estratificado por conglomerados, con una estratificación constante de 50 centros en cada una de las Comunidades Autónomas. De ellos, un $48.3 \%$ son mujeres, y la mayoría tienen entre 50-55 años (un 47.4\%), uno de cada tres tiene menos de 5 años de experiencia como director (el $34.5 \%$ ) y el $70.1 \%$ ha recibido formación específica para ejercer el cargo.

\section{Materiales}

Esta investigación utilizó diversos instrumentos utilizados. En primer lugar, cuatro pruebas de desempeño, una para cada una de las cuatro competencias evaluadas: Competencia en Comunicación Lingüística (fiabilidad de ,861), Competencia Matemática (,800), Competencia en el Conocimiento y la Interacción con el Mundo Físico (,854) y Competencia Social y Ciudadana (,856). En segundo lugar, un cuestionario dirigido a estudiantes y otro a familias, a partir del cual se elaboró el índice ISEC. Y, por último un cuestionario para directivos, del cual se obtienen los datos sobre las características de la escuela, del directivo y de la distribución del tiempo.

\section{Análisis}

Para conseguir los objetivos se utilizó una variedad de análisis estadísticos. En primer lugar, estadísticos descriptivos, con los que se estimó el tiempo de dedicación de los directivos y su distribución; en segundo término, un análisis de regresión lineal para determinar los factores asociados a la distribución; y, por último, los modelos multinivel de dos niveles (alumno y escuela) para estudiar la incidencia de la distribución del tiempo sobre el desempeño de los estudiantes y el análisis de clúster para hacer tipologías de directivos en función de cómo distribuyen su tiempo.

\section{Resultados}

\section{Incidencia de la distribución del tiempo de los directivos sobre el desempeño de los estudiantes}

El primero de los objetivos planteados es determinar la incidencia de la distribución del tiempo de los directivos en el desempeño de los estudiantes. Para conseguirlo, en primer lugar, se establecieron tres tipologías de directivos en función de cómo distribuyen su tiempo. Para ello se realizó un Análisis de Conglomerados bi-etápico. Del mismo se obtienen tres grupos de directivos en función de cómo distribuyen su tiempo (Tabla 2):

- Directivo A, predominan las tareas administrativas internas, a las que dedica un promedio del $43 \%$ de su tiempo global dedicado a las funciones directivas. Está compuesto por 234 directores y directoras, un $26,7 \%$ de la muestra.

- Directivo B, definido por su alta dedicación a tareas relacionadas con el currículo y la enseñanza general del centro, concretamente un $47 \%$ del tiempo de promedio. Conformado por 243 directivos, un 27,7\% de la muestra.

- Directivo C, determinado por su menor dedicación a tareas administrativas internas y a tareas relacionadas con el currículo, y mayor a las relacionadas con las peticiones de las administraciones y con las reuniones. De los directores y directoras, 399 de la muestra pertenecen a esta tipología (un 45,5\%). 
Tabla 2 - Conformación de las tipologías de directores: Centros de los conglomerados finales resultantes del análisis del análisis de conglomerado

\begin{tabular}{lccc}
\multicolumn{1}{c}{ Tareas } & Directivo & Directivo & Directivo \\
& A & B & 18 \\
\hline Tareas Administrativas internas & 43 & 22 \\
Tareas rel. con el currículo y la enseñanza & 24 & 14 & 22 \\
Tareas rel. con las peticiones de las Administraciones & 12 & 22 \\
Tareas rel. con reuniones con el profesorado, las familias... & 16 & 19 & 28 \\
\hline Número de casos & 234 & 243 & 399 \\
\hline
\end{tabular}

Fuente: Elaboración propia a partir de los datos de la Evaluación General Diagnóstica de Educación Primaria de 2009 (Instituto de Evaluación, 2010)

El segundo paso es ver si los estudiantes de escuelas con directivos de uno u otro tipo tiene diferentes resultados académicos en las cuatro variables de estudio: Competencia Lingüística, Competencia Matemática, Competencia Científica y Competencia Social y Ciudadana. Para ello, y dado que el rendimiento de los estudiantes y la tipología de directivo pertenecen a unidades de análisis diferentes, se utilizarán Modelos Multinivel de dos niveles (estudiante y escuela).

Los resultados de los cuatro procesos de modelado multinivel se muestran en las tablas 2 a 5 . De su estudio se pueden obtener algunas ideas previas. En primer lugar, que tanto el ISEC (Índice Socio-económico y Cultural) del estudiante como de la escuela, aparecen en los cuatro modelos ajustados y finales. Ello indica que están relacionados con el desempeño y que cumplen perfectamente su función de ajuste. Es más, aunque no sea el objeto de este estudio, se observa que por cada desviación típica que aumente el Î́ndice Socio Económico y Cultural (ISEC) del alumno, su rendimiento aumentará una media de 24 puntos (según la materia). La segunda variable que aparece en los modelos es la titularidad del centro. Según estos datos, los estudiantes de centros privados, controlado el ISEC, obtienen peores resultados que los públicos, excepto en competencia Social y Ciudadana que no hay diferencias. Lo más importante es que cumple su función de ajuste y con su incorporación se controla su posible injerencia en el modelo. El género del estudiante y la preescolarización, sin embargo, y a pesar de lo previsto, no realizan una aportación significativa en ninguno de los cuatro modelos.

Centrándonos en los objetivos de esta investigación, los modelos finales nos aportan datos muy interesantes. Para las cuatro variables de producto, los datos muestran que el coeficiente de la variable "pertenecer al grupo de directores B ", realiza una aportación significativa en tres de los cuatro modelos. Ello implica que los estudiantes cuyos directores o directoras pertenecen al grupo B, es decir, que dedican más tiempo a temas curriculares, obtienen mejores resultados que aquellos cuyos directivos se centran en otras actividades (controlando el efecto del ISEC de la familia del estudiante, del ISEC de la escuela y la titularidad del centro).

Más concretamente, los estudiantes cuyos directivos se dedican fundamentalmente a temas curriculares obtienen:

- 4,69 puntos más en Competencia Lingüística (Tabla 2);

- 5,15 puntos más en Competencia Matemática (Tabla 3); y

- 6,66 puntos más en Competencia Científica (Tabla 4).

Sin embargo, como se ha comentado, no parece incidir en la Competencia Social y Ciudadana (Tabla 3). 
Murillo, F. Javier \& Hernández-Castilla, Reyes (2015). Liderazgo para el aprendizaje: ¿Qué tareas de los directores y directoras escolares son las que más inciden en el aprendizaje de los estudiantes?. RELIEVE, 21 (1), art. 1. DOI: $\underline{10.7203 / \text { relieve.21.1.5015 }}$

Tabla 3 - Resultados de los modelos multinivel para Competencia Lingüística

\begin{tabular}{|c|c|c|c|}
\hline & Modelo Nulo & $\begin{array}{l}\text { Modelo } \\
\text { ajustado }\end{array}$ & Modelo Final \\
\hline & B (ee) & B (ee) & B (ee) \\
\hline \multicolumn{4}{|l|}{ Parte fija } \\
\hline Intercepto & $500,44(1,40)$ & $503,59(1,32)$ & $502,59(1,14)$ \\
\hline ISEC & & $25,91(, 65)$ & $25,91(, 65)$ \\
\hline IISEC-Escuela & & $30,71(2,41)$ & $31,02(2,41)$ \\
\hline Género & & NS & NS \\
\hline Preescolarización & & NS & NS \\
\hline Titularidad (público-privado) & & $-5,57(2,47)$ & $-5,64(2,47)$ \\
\hline \multicolumn{4}{|l|}{ Variables de proceso } \\
\hline Directivo A: centrado en tareas de administración interna & & & NS \\
\hline Directivo B: centrado en temas curriculares & & & $4,69(2,32)$ \\
\hline $\begin{array}{l}\text { Directivo C: centrado en reuniones y requisitos de la } \\
\text { Administración }\end{array}$ & & & NS \\
\hline \multicolumn{4}{|l|}{ Parte aleatoria } \\
\hline Entre escuelas & $1411,33(82,64)$ & $605,51(43,02)$ & $602,62(43,02)$ \\
\hline Entre alumnos & $8352,81(73,38)$ & $7864,81(69,65)$ & $7864,41(69,56)$ \\
\hline
\end{tabular}

Nota: NS Aportación significativa con $\alpha=, 05$.

Tabla 4 Resultados de los modelos multinivel para la Competencia Matemática

\begin{tabular}{|c|c|c|c|}
\hline & Modelo Nulo & $\begin{array}{c}\text { Modelo } \\
\text { ajustado }\end{array}$ & Modelo Final \\
\hline & B (ee) & B (ee) & B (ee) \\
\hline \multicolumn{4}{|l|}{ Parte fija } \\
\hline Intercepto & $500,58(1,39)$ & $503,54(1,35)$ & $502,63(1,44)$ \\
\hline ISEC & & $23,82(, 65)$ & $23,81(, 65)$ \\
\hline IISEC-Escuela & & $29,48(2,46)$ & $30,97(2,46)$ \\
\hline Género & & NS & - \\
\hline Preescolarización & & NS & - \\
\hline Titularidad (público-privado) & & $-5,65(2,63)$ & $-5,68(2,63)$ \\
\hline \multicolumn{4}{|l|}{ Variables de proceso } \\
\hline Directivo A: centrado en tareas de administración Interna & & & NS \\
\hline Directivo B: centrado en temas curriculares & & & $5,14(2,36)$ \\
\hline $\begin{array}{l}\text { Directivo C: centrado en reuniones y requisitos de la } \\
\text { Administración }\end{array}$ & & & NS \\
\hline \multicolumn{4}{|l|}{ Parte aleatoria } \\
\hline Entre escuelas & $1346,51(80,30)$ & $631,54(44,77)$ & $628,00(44,75)$ \\
\hline Entre alumnos & $8549,73(75,12)$ & $8125,27(71,96)$ & $8124,85(71,96)$ \\
\hline
\end{tabular}

Nota: NS Aportación significativa $\operatorname{con} \alpha=, 05$. 
Tabla 5 - Resultados de los modelos multinivel para la Competencia Científica

\begin{tabular}{|c|c|c|c|}
\hline & Modelo Nulo & Modelo ajustado & Modelo Final \\
\hline & B (ee) & B (ee) & B (ee) \\
\hline \multicolumn{4}{|l|}{ Parte fija } \\
\hline Intercepto & $500,49(1,53)$ & $504,33(1,67)$ & $504,32(1,79)$ \\
\hline ISEC & & $25,04(, 63)$ & $25,04(, 63)$ \\
\hline IISEC-Escuela & & 24,93 $(2,99)$ & $25,36(2,98)$ \\
\hline Género & & NS & - \\
\hline Preescolarización & & NS & - \\
\hline Titularidad (público-privado) & & $-6,70(3,23)$ & $-7,73(3,24)$ \\
\hline \multicolumn{4}{|l|}{ Variables de proceso } \\
\hline Directivo A: centrado en tareas de administración interna & & & NS \\
\hline Directivo B: centrado en temas curriculares & & & $6,66(2,92)$ \\
\hline $\begin{array}{l}\text { Directivo C: centrado en reuniones y requisitos de la } \\
\text { Administración }\end{array}$ & & & NS* \\
\hline \multicolumn{4}{|l|}{ Parte aleatoria } \\
\hline Entre escuelas & $1769,38(99,00)$ & $1149,13(69,07)$ & $1141,75(68,9$ \\
\hline Entre alumnos & $7965,36(70,03)$ & $7505,24(66,50)$ & $7505,07(66,4$ ؛ \\
\hline
\end{tabular}

Tabla 6 - Resultados de los modelos multinivel para Competencia Social y Ciudadana

\begin{tabular}{|c|c|c|c|}
\hline & Modelo Nulo & $\begin{array}{l}\text { Modelo } \\
\text { ajustado }\end{array}$ & Modelo Final \\
\hline & B (ee) & B (ee) & B (ee) \\
\hline \multicolumn{4}{|l|}{ Parte fija } \\
\hline Intercepto & $500,54(1,42)$ & $501,63(1,16)$ & $501,63(1,16)$ \\
\hline ISEC & & 23,19 (,64) & 23,19 (,64) \\
\hline IISEC-Escuela & & $23,22(2,34)$ & $23,22(2,34)$ \\
\hline Género & & NS & - \\
\hline Preescolarización & & NS & - \\
\hline Titularidad (público-privado) & & NS & - \\
\hline \multicolumn{4}{|l|}{ Variables de proceso } \\
\hline Directivo A: centrado en tareas de administración interna & & & NS \\
\hline Directivo B: centrado en temas curriculares & & & NS \\
\hline $\begin{array}{l}\text { Directivo C: centrado en reuniones y requisitos de la } \\
\text { Administración }\end{array}$ & & & NS \\
\hline \multicolumn{4}{|l|}{ Parte aleatoria } \\
\hline Entre escuelas & $1467(84,83)$ & $888,46(56,91)$ & $888,46(56,91)$ \\
\hline Entre alumnos & $8312,95(73,12)$ & $7921,13(70,27)$ & $7921,13(70,27)$ \\
\hline
\end{tabular}

Nota: NS Aportación significativa con $\alpha=, 05$.

\section{Factores asociados a la distribución del tiempo de los directivos}

Establecida la importancia de la distribución del tiempo de los directores y directoras de escuelas de Primaria en España en el desempeño de los estudiantes, el segundo objetivo del estudio es determinar qué factores están asociados a la distribución del tiempo de los directivos antes descrita. Se estudian ocho variables de dos tipos diferentes, características del centro y del directivo. Las características del centro son: Titularidad (público-privado), tamaño, y nivel socioeconómico y cultural de las familias de los 
estudiantes; y las características del directivo analizadas son: el género, la edad, la experiencia en tareas directivas y la satisfacción con la escuela.

Veamos, en primer lugar, cómo es la distribución del tiempo de los directivos según su propia opinión (Figura 3):

- Tareas administrativas internas: 26,66\% del tiempo.

- Tareas relacionadas con el currículo y la enseñanza general en el centro: 29,25\%.

- Tareas relacionadas con las peticiones de las Administraciones Local, Autonómica y Central: 19,96\%.
- Tareas relacionadas con las reuniones con el profesorado o las familias: $22,21 \%$ del tiempo.

- Otras tareas: 4,92\% del tiempo.

De esta forma, los directivos españoles de centros de Primaria, en una hipotética jornada laboral de 8 horas en la que no tengan obligaciones lectivas, dedican 3 horas y 43 minutos a tareas de carácter administrativo, 2 horas y 20 minutos a tareas relacionadas con el currículo, 1 hora y 46 minutos se dedica a reuniones y los 23 minutos restantes a otras actividades.

Figura 3 - Distribución del tiempo de los directivos escolares de la educación primaria en España
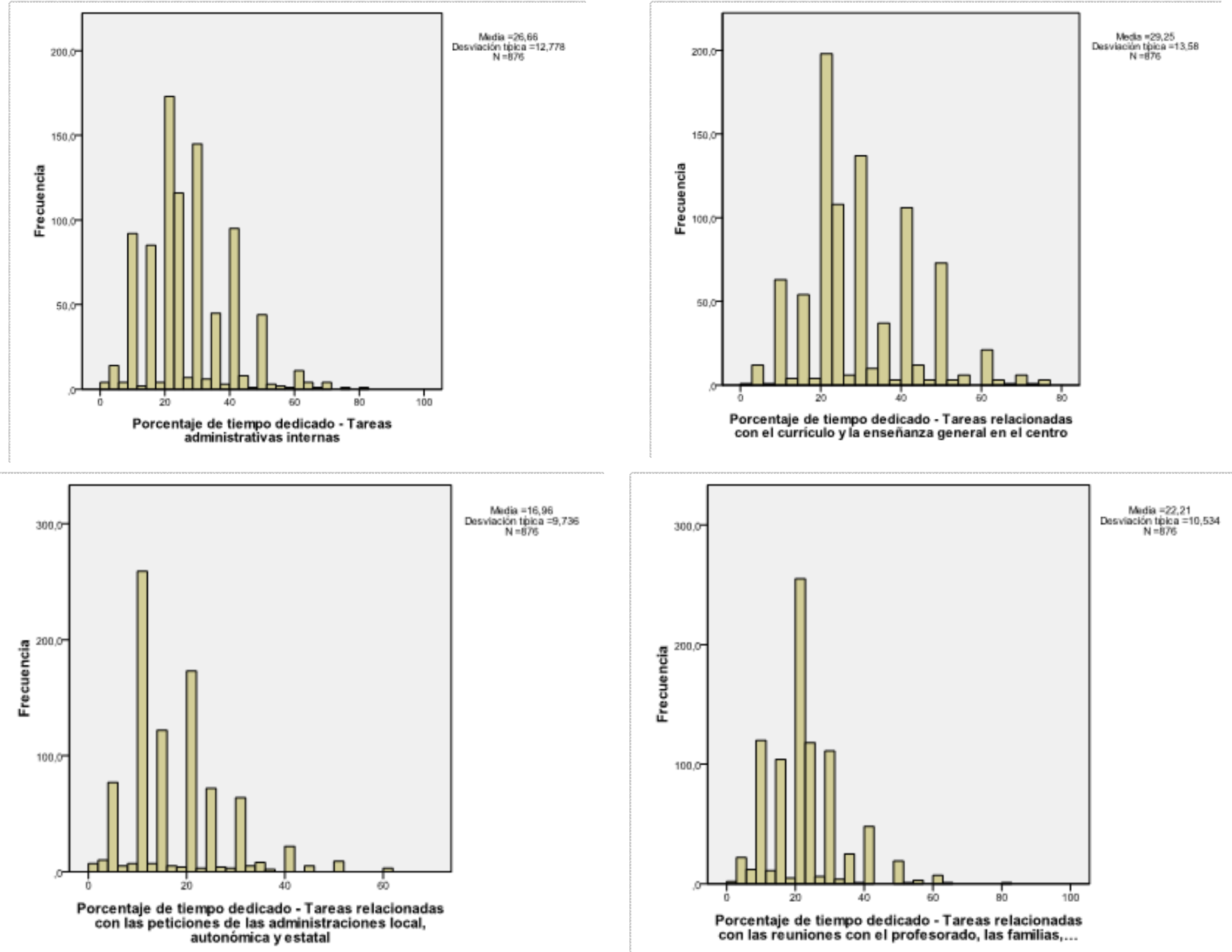

Fuente: Elaboración propia a partir de los datos de la Evaluación General Diagnóstica de Educación Primaria de 2009 (Instituto de Evaluación, 2010) 
Llama la atención la gran dispersión en la dedicación a cada una de estas tareas de los directores consultados. Más allá de la normal variabilidad en la actuación de cada directivo, no sería muy arriesgado pensar que algunos directivos han exagerado sus respuestas (Tabla 7).

Tabla 7 - Distribución de los directivos en función del porcentaje del tiempo que dedican a cada una de las tareas

\begin{tabular}{lllllllll}
\hline \multicolumn{1}{c}{ Tareas } & $0-10$ & $11-20$ & $21-30$ & $31-40$ & $41-50$ & $51-60$ & $51-70$ & $71-$ \\
\hline Tareas administrativas internas & 12,62 & 29,00 & 31,72 & 17,14 & 6,19 & 2,13 &, 97 &, 22 \\
Tareas rel. con el currículo y la enseñanza & 8,33 & 29,32 & 29,34 & 17,73 & 10,41 & 3,30 & 1,11 &, 46 \\
$\begin{array}{l}\text { Tareas rel. con las peticiones de las } \\
\quad \text { Administración }\end{array}$ & 40,94 & 36,29 & 16,63 & 4,30 & 1,50 &, 33 &, 00 &, 00 \\
$\begin{array}{l}\text { Tareas relacionadas con reuniones } \\
\text { Otras tareas }\end{array}$ & 17,12 & 42,84 & 27,55 & 8,96 & 2,07 & 1,08 &, 28 &, 10 \\
& 90,54 & 7,16 & 1,09 &, 67 &, 43 &, 11 &, 00 &, 00 \\
\hline
\end{tabular}

Fuente: Elaboración propia a partir de los datos de la Evaluación General Diagnóstica de Educación Primaria de 2009 (INEE, 2011).

Para determinar qué factores están asociados a la distribución del tiempo de los directivos se estudian ocho variables de dos tipos diferentes, características del centro y del directivo. Las características del centro son: Titularidad (público-privado), tamaño, y nivel socio-económico y cultural de las familias de los estudiantes; y las características del directivo analizadas son: el género, la edad, la experiencia en tareas directivas y la satisfacción con la escuela. De ellas, cinco han mostrado relación con algún aspecto de la distribución del tiempo: la titularidad del centro y su tamaño; y el género del directivo, su edad y formación específica.

Efectivamente, los resultados de los análisis de regresión lineal, uno para cada aspecto de la distribución del tiempo (Tabla 8), muestran que:

- Los directivos de centros privados dedican 5,7 puntos porcentuales menos de tiempo a tareas administrativas, 7,18 más a tareas relacionadas con el currículo y la enseñanza, 2 menos a tareas relacionadas con las peticiones de las administraciones; 1,7 más a tareas asociadas con reuniones con profesorado o familias.

- Los directivos de centros más grandes dedican menos tiempo a tareas relacionadas con el currículo y más con reuniones.

- Las directivas mujeres dedican menos porcentaje de tiempo a tareas administrativas internas y más a reuniones con profesorado y familias.

- Los directivos de más edad invierten más porcentaje de tiempo en tareas relacionadas con el currículo.

- Los directivos que han recibido formación específica para la dirección dedican más tiempo a las tareas relacionadas con las reuniones con padres y estudiantes. 
Murillo, F. Javier \& Hernández-Castilla, Reyes (2015). Liderazgo para el aprendizaje: ¿Qué tareas de los directores y directoras escolares son las que más inciden en el aprendizaje de los estudiantes?. RELIEVE, 21 (1), art. 1. DOI: $\underline{10.7203 / \text { relieve.21.1.5015 }}$

Tabla 8 - Factores asociados a la distribución del tiempo de los directivos de centros españoles de educación primaria. Resultados de los Análisis de Regresión Lineal

\begin{tabular}{|c|c|c|c|c|c|}
\hline VD & VI & B & E.E. & $\mathbf{T}$ & Sig. \\
\hline \multirow{3}{*}{$\begin{array}{l}\text { Tareas Administrativas } \\
\text { internas }^{\text {a }}\end{array}$} & Intercepto & 30,365 & ,654 & 46,419 & ,000 \\
\hline & Centro (Público/Privado) & $-5,765$ & ,945 & $-6,102$ & ,000 \\
\hline & Género directivo (Hombre/Mujer) & $-3,918$ & ,868 & $-4,512$ & 000 \\
\hline \multirow{4}{*}{$\begin{array}{l}\text { Tareas relacionadas } \\
\text { con el currículo y } \\
\text { enseñanza }{ }^{b}\end{array}$} & Intercepto & 27,469 & 2,131 & 12,888 & 000 \\
\hline & Tamaño centro &,- 176 & ,023 & $-7,743$ & 000 \\
\hline & Centro (Público/Privado) & 7,182 & 1,111 & 6,462 &, 000 \\
\hline & Edad directivo & 1,756 &, 542 & 3,242 & 001 \\
\hline \multirow{3}{*}{$\begin{array}{l}\text { Tareas relacionadas } \\
\text { con las peticiones de } \\
\text { las Administraciones }{ }^{\mathrm{c}}\end{array}$} & Intercepto & 17,076 &, 514 & 33,193 & 000 \\
\hline & Centro (Público/Privado) & $-2,058$ & ,743 & $-2,770$ & ,006 \\
\hline & Género Directivo(Hombre/Mujer) & 1,374 & ,683 & 2,013 & 044 \\
\hline \multirow{5}{*}{$\begin{array}{l}\text { Tareas relacionadas } \\
\text { con reuniones con el } \\
\text { profesorado, las } \\
\text { familias... d }\end{array}$} & Intercepto & 12,4976 & 1,180 & 10,588 & 000 \\
\hline & Tamaño centro & ,120 & ,018 & 6,822 & ,000 \\
\hline & Género directivo (Hombre/Mujer) & 3,350 & ,702 & 4,771 & ,000 \\
\hline & Formación cargo directivo & 1,799 & ,766 & 2,350 & ,019 \\
\hline & Centro (Público/Privado) & 1,850 & 804 & 2,302 & 022 \\
\hline
\end{tabular}

(a) Variables excluidas del modelo: ISEC-escuela, Tamaño del centro, Edad del directivo, Experiencia en tareas directivas, Formación cargo directivo, Satisfacción del directivo.

(b) Variables excluidas del modelo: ISEC-escuela, Género del directivo, Experiencia en tareas directivas, Formación cargo directivo, Satisfacción del directivo.

(c) Variables excluidas del modelo: ISEC-escuela, Tamaño del centro, Edad del directivo, Experiencia en tareas directivas, Formación cargo directivo, Satisfacción del directivo.

(d) Variables excluidas del modelo: ISEC-escuela, Edad del directivo, Experiencia en tareas directivas, Formación cargo directivo, Satisfacción del directivo.

Fuente: Elaboración propia a partir de los datos de la Evaluación General Diagnóstica de Educación Primaria de 2009 (Instituto de Evaluación, 2010).

Veamos con detalle esos resultados, analizando cómo es la distribución del tiempo de los directivos en función de esas cinco variables explicativas.

La titularidad del centro, como hemos visto, incide en la distribución del tiempo de sus directivos. Efectivamente, los datos indican que los directivos de centros públicos dedican más tiempo a tareas administrativas, tanto internas como relacionadas con las peticiones de las Administraciones, y menos-a reunirse con docentes $\mathrm{y}$ familias $\mathrm{y}$ a tareas relacionadas con el currículo y la enseñanza (Tabla 9).

Tabla 9- Distribución del tiempo de los directivos de centros españoles de educación primaria en función de la titularidad del centro

\begin{tabular}{lccc}
\multicolumn{1}{c}{ Tareas } & Centro Público & $\begin{array}{c}\text { Centro privado } \\
\text { concertado }\end{array}$ & $\begin{array}{c}\text { Centro privado no } \\
\text { concertado }\end{array}$ \\
\hline Tareas administrativas internas & 28,51 & 22,38 & 22,73 \\
$\begin{array}{l}\text { Tareas relacionadas con el currículo y la } \\
\text { enseñanza }\end{array}$ & 28,19 & 31,63 & 32,27 \\
Tareas rel, con las peticiones de las & 17,61 & 15,73 & 11,36 \\
$\quad$ Administración & 20,82 & 25,13 & 31,36 \\
Tareas relacionadas con reuniones & 4,87 & 5,13 & 2,27 \\
Otras tareas & & & \\
\hline
\end{tabular}

Fuente: Elaboración propia a partir de los datos de la Evaluación General Diagnóstica de Educación Primaria de 2009 (Instituto de Evaluación, 2010). 
También el tamaño del centro parece incidir en la distribución del tiempo de los directores (independientemente de la titularidad del centro). De esta forma, cuanto más grande es el centro, menos tiempo se dedican los directores y directoras a temas relacionados con el currículo y la enseñanza y más a tener reuniones con profesores y familias.

Esta relación puede ser observada en la Tabla 10 donde se ha agrupado a los centros en tres bloques en función de su tamaño: pequeños, con menos de 29 docentes, de tamaño medio, entre 29 y 42 docentes, y grandes, más de 42 maestros y maestras. Los puntos de corte se corresponden a los percentiles 33 y 66, de tal forma que el número de centros que componen cada grupo es similar, unos 300 . Allí se observa que mientras que el tiempo dedicado a las tareas administrativas se mantiene bastante estable, en los centros pequeños los directores y directoras dedican más tiempo a tareas relacionados con el currículo y menos a las reuniones. En los grandes, por el contrario, la gran cantidad de tiempo dedicado a las reuniones es compensada por el poco dedicado a tareas relacionadas con el currículo y la enseñanza general del centro.

Tabla 10 - Distribución del tiempo de los directivos de los centros españoles de educación primaria en función del tamaño del centro

\begin{tabular}{lccc}
\hline \multicolumn{1}{c}{ Tareas } & $\begin{array}{c}\text { Pequeño (menos } \\
\text { de 29 docentes) }\end{array}$ & $\begin{array}{c}\text { Medio (entre 29 } \\
\text { y 42 docentes) }\end{array}$ & $\begin{array}{c}\text { Grande (más de } \\
\text { 42 docentes) }\end{array}$ \\
\hline Tareas administrativas internas & 26,44 & 28,60 & 25,11 \\
Tareas rel. con el currículo y la enseñanza & 33,66 & 27,36 & 26,21 \\
$\begin{array}{l}\text { Tareas rel, con las peticiones de las } \\
\text { Administraciones }\end{array}$ & 16,40 & 17,76 & 17,06 \\
$\begin{array}{l}\text { Tareas rel. con reuniones con el profesorado, } \\
\text { las familias... }\end{array}$ & 19,16 & 21,56 & 26,21 \\
Otras tareas & 4,33 & 4,73 & 5,41 \\
\hline
\end{tabular}

Fuente: Elaboración propia a partir de los datos de la Evaluación General Diagnóstica de Educación Primaria de 2009 (Instituto de Evaluación, 2010).

Los Análisis de Regresión Lineal antes mostrados indican que, controlando otras variables, los directores distribuyen su tiempo de forma diferente a las directoras. Según los datos (tabla 11), las mujeres dedican menos a cuestiones administrativas, el $41,8 \%$ frente al
45,23 de los hombres, especialmente a administración interna, y más a tener reuniones con el profesorado. Ello, en coherencia con otras investigaciones, muestra estilos de dirección diferentes entre hombres y mujeres.

Tabla 11 - Distribución del tiempo de los directivos de centros españoles de educación primaria en función de su género

\begin{tabular}{lrc}
\hline \multicolumn{1}{c}{ Tareas } & Hombre & Mujer \\
\hline Tareas administrativas internas & 28,83 & 24,33 \\
Tareas rel. con el currículo y la enseñanza & 28,80 & 29,71 \\
Tareas rel. con las peticiones de las Administraciones & 16,44 & 17,56 \\
Tareas rel. con reuniones con el profesorado, las familias & 20,64 & 23,90 \\
Otras tareas & 5,30 & 4,50 \\
\hline
\end{tabular}

Fuente: Elaboración propia a partir de los datos de la Evaluación General Diagnóstica de Educación Primaria de 2009 (Instituto de Evaluación, 2010). 
Otro factor que ha mostrado su incidencia en la distribución del tiempo es la edad del directivo. La variable "Experiencia en funciones directivas" también está relacionada con la distribución; concretamente la correlación entre el porcentaje de tiempo dedicado a temas curriculares y la experiencia como director o directora es estadísticamente significativo con un $\alpha=, 05(, 08)$. Sin embargo, conjuntamente con la edad, esta parece explicar en mayor medida las diferencias.

Así, según estos datos, los directores o directoras de mayor edad dedican más tiempo a tareas relacionadas con el currículo y, como consecuencia, menos a otras actividades (Tabla 12). De modo que un director o directora de menos de 30 años dedica el 22,0\% de su tiempo a tareas relacionadas con el currículo y un 52,0\% a tareas relacionadas con la administración, mientras que los de más de 60 años, esas mismas cifras son considerablemente diferentes, de $21,4 \%$ y de $42,1 \%$, respectivamente. La edad, por tanto, marca diferencias importantes en el estilo de dirección.

Tabla 12 - Distribución del tiempo de los directivos de centros españoles de educación primaria en función de su edad

\begin{tabular}{|c|c|c|c|c|c|}
\hline Tareas & $\begin{array}{l}\text { Menos de } \\
30 \text { años }\end{array}$ & $\begin{array}{l}\text { 30-39 } \\
\text { años }\end{array}$ & $\begin{array}{l}40-49 \\
\text { años }\end{array}$ & $\begin{array}{l}50-59 \\
\text { años }\end{array}$ & $\begin{array}{l}60 \text { o } \\
\text { más }\end{array}$ \\
\hline Tareas administrativas internas & 30,00 & 27,07 & 27,21 & 26,35 & 25,22 \\
\hline Tareas rel. con el currículo y la enseñanza & 22,00 & 27,35 & 27,87 & 30,29 & 31,40 \\
\hline $\begin{array}{l}\text { Tareas rel. con las peticiones de las } \\
\text { Administraciones }\end{array}$ & 22,00 & 17,04 & 17,09 & 16,88 & 16,90 \\
\hline $\begin{array}{l}\text { Tareas rel. con reuniones con el profesorado, } \\
\text { familias... }\end{array}$ & 20,00 & 22,65 & 22,88 & 21,51 & 23,35 \\
\hline Otras tareas & 6,00 & 5,89 & 4,95 & 4,99 & 3,13 \\
\hline
\end{tabular}

Fuente: Elaboración propia a partir de los datos de la Evaluación General Diagnóstica de Educación Primarias de 2009 (Instituto de Evaluación, 2010)

El último factor que, según los datos, está relacionado con la distribución del tiempo de los directores es el haber recibido o no formación para cargos directivos. Recordemos que el $70,1 \%$ de los directores y directores afirman que sí la recibieron. Los datos indican que este hecho incide significativamente sólo en el tiempo dedicado a tareas relacionadas con reuniones con el profesorado y familias. Los directores con formación dedican más tiempo a ellas (Tabla 13).

Tabla 13 - Distribución del tiempo de los directivos de centros españoles de educación primaria en función de si ha recibido formación para los cargos directivos

\begin{tabular}{lcc}
\multicolumn{1}{c}{ Tareas } & Sin formación & Con formación \\
\hline Tareas administrativas internas & 27,06 & 26,49 \\
Tareas rel. con el currículo y la enseñanza & 30,24 & 28,85 \\
Tareas rel. con las peticiones de las Administraciones & 16,79 & 17,04 \\
Tareas rel. con reuniones con el profesorado, las familias... & 21,08 & 22,68 \\
Otras tareas & 4,84 & 4,94 \\
\hline
\end{tabular}

Fuente: Elaboración propia a partir de los datos de la Evaluación General Diagnóstica de Educación Primarias de 2009 (INEE, 2011). 


\section{Discusión y conclusiones}

Esta investigación se enmarca en la fértil línea de estudio que busca profundizar en el mayor conocimiento de la distribución del tiempo de los directores y en su incidencia en el centro escolar. Los resultados encontrados tienen la potencialidad de haber sido obtenidos a partir de una amplia muestra de directores y directoras, por lo que resultan representativos del conjunto de España, por lo que su fiabilidad está garantizada.

Atendiendo a cada uno de los tres objetivos, la investigación ha ofrecido, en primer lugar, datos claros sobre la distribución del tiempo de los directores de los centros de Educación Primaria. A grandes rasgos, los resultados muestran que dedican la mayor parte del tiempo a tareas administrativas de todo tipo (un 46\%), un $29 \%$ a tareas de carácter pedagógico y curricular y un $22 \%$ a tener reuniones.

El único estudio anterior de iguales características y con directivos españoles fue desarrollado con datos de hace 15 años (Murillo y Barrio, 1999). Dicho trabajo encontró igualmente que los directores y directoras dedicaban una buena parte de su tiempo tareas burocráticas (en este caso un $36,4 \%$ del tiempo) y menos a tareas de carácter curricular (un 26,7\%). Dadas las diferencias en las tareas consideradas en estos dos trabajos no es posible deducir si estas diferencias son debidas a los cambios acontecidos 15 años después. Por ello, resulta difícil sacar conclusiones sobre de los procesos producidos. En el trabajo del TIMSS se encontró que el 32\% del tiempo se dedican a tareas administrativas. Datos análogos a los hallados en este estudio, pero considerando sólo las tareas administrativas internas.

Esta explotación especial también encontró que el género y la edad inciden en la distribución del tiempo de los directivos. Así, las directoras mujeres, y los de más edad dedican un menor porcentaje a actividades administrativas $\mathrm{y}$, con ello un mayor porcentaje de tiempo a tareas relacionadas con el currículum y la enseñanza y así como a tener reuniones con docentes y familias. Estos resultados son coherentes con otros trabajos (p.e. Murillo y Barrio, 1999; Murillo y Román, 2013), y muestran que hombre y mujer tienen formas de liderar el centro diferentes, así como los directores de mayor edad frente a los más jóvenes.

Respondiendo al tercer objetivo, el más relevante desde un punto de vista aplicado, la investigación ha encontrado evidencias que podrían contribuir a hacer mejores directores. Aquellos que consiguen que sus estudiantes aprendan, son los que más tiempo dedican a tareas curriculares. Este resultado coincide con el hallado por Murillo y Barrio (1999), con la misma metodología, y por Murillo y Román (2013). El meta-análisis de Robinson, Lloyd, y Rowe (2008) con estudios sobre la relación entre el liderazgo escolar y resultados de los estudiantes, llegaron a la misma concusión: los directores con un estilo Pedagógico tienen una incidencia positiva sobre el rendimiento de los estudiantes.

Relacionando los tres objetivos entre sí, se encuentran interesantes conclusiones. De un lado, son mejores directores las mujeres, las personas de más edad y las que tiene una mayor formación como directivos. Lo son dado que dedican más tiempo a tareas pedagógicas y menos a las administrativas. $\mathrm{Y}$ de otro, los centros más pequeños y los de titularidad privada posibilitan una mejor dirección en la medida en que ello permite que los directores y directoras se dediquen más a tareas de carácter pedagógico.

Este estudio cuenta como gran potencialidad el manejo de una gran cantidad de datos obtenidos con un muestreo estadísticamente representativo para el total de la nación; por lo que su validez externa, su capacidad de generalización, está comprobada. Sin embargo, comparte con otros estudios la limitación de que los datos de la opinión de los directores sobre su tiempo, no la realidad de dicha distribución. Para este estudio en particular es una limitación que las categorías de distribución del tiempo sean diferentes a las usadas en otros trabajos. Sería positivo que el Instituto de Evaluación mantuviera esas 
preguntas en futuras evaluaciones $y$ las incluyera en las evaluaciones diagnósticas de Secundaria. Con ello se obtendría una interesantísima evolución de estos datos en España.

Las aplicaciones prácticas de este estudio son inmediatas. Por un lado, dar el claro mensaje a los directores y directoras de que el tiempo que más incide en la mejora de los aprendizajes es aquel que está dedicado a cuestiones pedagógicas. Si en algo hay que delegar es en las tareas administrativas.

Y, por otro, a las Administraciones; los datos son claros en demostrar que los directores y directoras dedican demasiado tiempo a las tareas administrativas, tanto internas como externas; y eso es en gran medida por las demandas de las Administraciones. Ayudar a los directivos a que se ocupen de lo pedagógico es también contribuir a la mejora de la calidad de la educación.

La investigación ha demostrado que el trabajo del director o directora incide en el aprendizaje de los estudiantes; preocuparse por en que los directivos se dediquen a "lo importante", a las cuestiones pedagógicas, es una estrategia más para mejorar la calidad de la educación. Si queremos buenos directores, necesitamos líderes pedagógicos, no gestores.

\section{Referencias}

Antúnez, S. (1993). La "dirección de sí mismo” en el uso racional del tiempo personal. En J. Gairín y S. Antúnez (coords.), Organización escolar. Nuevas aportaciones. Barcelona: P.P.U.

Armas, M. (1998). Dirección integral de Centros Educativos. Santiago de Compostela: Tórculo Ediciones.

Beauchamp, L. \& Parsons, J. (2014). Liderazgo instructivo en Alberta: Hallazgos de la investigación en cinco escuelas altamente eficaces. REICE. Revista Iberoamericana sobre Calidad, Eficacia y Cambio en Educación, 12(4e), 89-98. Recuperado de http://www.rinace.net/reice/numeros/arts/vol12nu m4e/art4.pdf

Burke, J.R. (1980). A study of similarities and differences in elementary principals' perceived allocation an ideal allocation of time. (Tesis doctoral). Florida State University. Tallahassee.

Buttram, J.L., Mead, H., Loftus, D. \& Wilson, J.O. (2008). Allocation of school leaders time. Annual Meeting of American Educational Research Association. Nueva York.

Eberts, R.W. \& Stone, J.A. (1988). Student achievement in public schools: Do principals make a difference? Economics of Education Review, 7, 291-299. DOI:10.1016/02727757(88)90002-7

Ghosey, L.W. (1987). Actual, estimated, and ideal time used by principals of sixty-four largest high school in Kansas. (Tesis doctoral) University of Kansas. Kansas.

Gimeno, J. (Coord.) (1995). La dirección de centros: análisis de tareas. Madrid: CIDE.

Goldring, E., Huff, J., May, H. \& Camburn, E. (2008). School context and individual characteristics: What influences principal practice? Journal of Educational Administration, 46, 332-352. DOI:10.1108/09578230810869275

Gordon, R.L. (1996). Relationships among secondary principal use of time, principal effectiveness, and student achievement. (Tesis doctoral). University of Missouri-Columbia. Columbia, Missouri.

Gorman, M.R. (1993). Time management strategies and the implications for instructional leadership of high school principals: A case study analysis. (Tesis doctoral). Widener University. Chester, Pennsylvania.

Hallet, J. (1985). The relationship between gender and school principal's allocation of time. (Tesis doctoral). University of Washington. Washington.

Helps, R. (1994). The allocation of non-contact time to deputy headteachers in Primary schools. School Organisation, 14(3), 243-246. DOI: $\underline{10.1080 / 0260136940140301}$

Horng, E.L., Klasik, D. \& Loeb, S. (2010). Principal's time use and school effectiveness. American Journal of Education, 116(4), 491-593. Recuperado de:http://www.jstor.org/stable/10.1086/653625

Instituto De Evaluación (2010). Evaluación General de Diagnóstico 2009. Educación Primaria. Madrid: Ministerio de Educación. 
Kmetz, J.T. \& Willower, D.J. (1982). Elementary school principals' work behavior. Educational Administration Quarterly, 18(4), 62-78. DOI:10.1177/0013161X82018004006

Larry, C. D. (2003). A study of time management use and preferred time management practices of middle and secondary school principals in selected southern states. (Tesis doctoral inédita). University of Alabama. Alabama.

Lee, M. \& Hallinger, P. (2012). National contexts influencing principals' time use and allocation: economic development, societal culture, and educational system, School Effectiveness and School Improvement, 23(4), 461-482. Recuperado de: http://www.ieairc.org/fileadmin/IRC_2010_papers/PIRLS/Lee_ Hallinger.pdf

López-Yáñez, J., García-Jiménez, E., OlivaRodríguez, N., Moreta-Jurado, B. \& Bellerín, A. (2014). El liderazgo escolar a través del análisis de la actividad diaria de los directores. REICE. Revista Iberoamericana sobre Calidad, Eficacia y Cambio en Educación. 12(5),61-78. Recuperado de http://www.rinace.net/reice/numeros/arts/vol12nu $\underline{\text { m5/art4.pdf }}$

Marshall, S.G. (2014). Cambio en el liderazgo educativo intermedio en Nueva Zelanda: el jamón en el sándwich. REICE. Revista Iberoamericana sobre Calidad, Eficacia $y$ Cambio en Educación, 12(4e),163-189. Recuperado de http://www.rinace.net/reice/numeros/arts/vol12nu m4e/art7.pdf

Martin, W.J. \& Willower, D.J. (1981). The managerial behavior of high school principals. Educational Administration Quarterly, 17(1), 69-90. DOI: 10.1177/0013161X8101700105

Martinko, M.J. y Gardner, W.L. (1990). Structured observation of managerial work: A replication and synthesis. Journal of Management Studies, 27, 329-357. DOI: 10.1111/j.14676486.1990.tb00250.x

May, H., Huff. J. y Goldring, E. (2012). A longitudinal study of principals' activities and student performance. School Effectiveness and School Improvement, 23(4), 417-439. DOI: $\underline{10.1080 / 09243453.2012 .678866}$

Mcpeake, J.E. (2007). The principalship: a study of the principal's time on task from 1960 to the twenty-first century. (Tesis doctoral). Marshall University Virginia. Virginia

Mullis, I.V.S., Martin, M. O. y Foy, P. (2008). TIMSS 2007. International Mathematics Report: findings from IEA. Trends in International Mathematics and Science Study at the Fourth and Eighth Grades. Chestnut Hill MA: TIMSS y PIRLS International Study Centre. Boston College.

Murillo, F.J. (2006). Una Dirección Escolar para el Cambio: del Liderazgo Transformacional al Liderazgo Distribuido, REICE. Revista Iberoamericana sobre Calidad, Eficacia $y$ Cambio en Educación, 4(4), 11-24. Recuperado de http://www.rinace.net/arts/vol4num4e/art2.pdf

Murillo, F.J. (Coord.) (2007). Investigación Iberoamericana sobre Eficacia Escolar. Bogotá: Convenio Andrés Bello. Recuperado dehttp://www.uam.es/personal_pdi/stmaria/jmuril lo/documentos/IIEE.pdf

Murillo, F.J. y Barrio, R. (1999). Análisis de la distribución del tiempo de los directivos de centros de enseñanza Primaria. Revista de Educación, 319,201-222. DOI: 10.4438/1988592X-0034-8082-RE

Murillo, F.J. y Hernández-Castilla, R. (2014). Liderando escuelas justas para la Justicia Social. Revista Internacional de Educación para la Justicia Social, 3(2), 17-37. Recuperado de:http://www.rinace.net/riejs/numeros/vol3num2/art1.htm

Murillo, F.J. y Román, M. (2013). La distribución del tiempo de los directores y las directoras de escuelas de Educación Primaria en América Latina y su incidencia en el desempeño de los estudiantes. Revista de Educación, 361, 141-170. DOI: 10-4438/1988-592X-RE-2011-361-138

Murillo, F.J., Barrio, R. y Pérez-Albo, M.J. (1999). La dirección escolar: análisis e investigación. Madrid: $\quad$ CIDE. Recuperado dehttp://www.uam.es/personal_pdi/stmaria/jmuril lo/recursos/Documentos/Murillo (1999).pdf

Murua, N.S.S., y Piédrola, J.J.G. (2013). Liderazgo transformacional, empoderamiento y aprendizaje: un estudio en Ciclos Formativos de Grado Superior. Revista de Educación, 362, 594622.DOI: 10.4438/1988-592X-RE-2013-362-243

Osborne, W. y Wiggins, T. (1989). Perceptions on tasks in the school principalship. Journal of Personnel Evaluation in Education, 2(3), 367- 


\section{$376 . \quad$ Recuperado de:http://link.springer.com/article/10.1007\%2FB F00139651?LI=true\#}

Padilla Carmona, M.T. (2008). Opiniones y experiencias en el desempeño de la dirección escolar de las mujeres directoras en Andalucía. RELIEVE,14(1) Recuperado de:http://www.uv.es/RELIEVE/v14n1/RELIEVE v14n1_1.htm

Rayfield, R. y Diamantes, T. (2004). Task analysis of the duties performed in Secondary School administration, Education, 124(4), 709-713. Recuperado de: http://eric.ed.gov/?id=EJ705778

Robinson, V.M.J., Lloyd, C.A. y Rowe, K.J. (2014). El impacto del liderazgo en los resultados de los estudiantes: Un análisis de los efectos diferenciales de los tipos de liderazgo. REICE. Revista Iberoamericana sobre Calidad, Eficacia y Cambio en Educación, 12(4e), 13-40. Recuperado de http://www.rinace.net/reice/numeros/arts/vol12nu m4e/art1.pdf

Silins, H. y Mulford, B. (2010). Reconceptualising school principalship that improves student outcomes. Journal of Educational Leadership, Policy and Practice, 25(2), 73-92.DOI:11108/09513541111100125

Spillane, J.P., Camburn, E.M. y Pareja, A. (2007). Taking a distributed perspective to the school principal's workday. Leadership and Policy in Schools, 6, 103-125. DOI: $\underline{10.1080 / 15700760601091200}$

Taylor, K.C. (2007). A study of principal's perceptions regarding time management. (Tesis doctoral). Kansas State University. Kansas.

Thorpe, A. (2014). Why the Leadership and Management Divide Matters in Education: the Implications for Schools and Social Justice.
Revista Internacional de Educación para la Justicia Social (RIEJS), 3(2), 199-212. Recuperado de: http://www.rinace.net/riejs/numeros/vol3num2/art10.htm

Walker, J. (2009). Reorganizing Leaders' Time: Does it Create Better Schools for Students? Comunicación presentada en el Annual Conference of the National Council of Professors of Educational Administration. San Antonio, Texas: San Antonio University.

Walker, A.D., Lee, M. y Bryant, D.A. (2014) School Effectiveness and School Improvement (2014): How much of a difference do principals make? An analysis of between-schools variation in academic achievement in Hong Kong public secondary schools, School Effectiveness and School Improvement: An International Journal of Research, Policy and Practice. DOI: $\underline{10.1080 / 09243453.2013 .875044}$

Wells, G.A. (1993). Instructional management behaviour, time management, and selected background variables of elementary school principals in Connecticut's urban school districts. (Tesis doctoral). University of Connecticut. Connecticut.

Wolcott, H.F. (1973). The man in the principal's office: An ethnography. San Francisco, CA: Holt, Rinehart and Winston.

\section{Nota}

Este estudio se ha realizado a partir de la explotación de bases de datos de acceso libre:

. http://www.mecd.gob.es/dctm/ievaluacion/basesde-datos/egd09.zip?documentId=0901e72b813c8ea6 - http://www.mecd.gob.es/inee/Bases-de-datos.html 


\begin{tabular}{|c|c|}
\hline Autor & To know more / Saber más \\
\hline \multicolumn{2}{|l|}{ Murillo, F. Javier (javier.murillo@uam.es). } \\
\hline \multicolumn{2}{|l|}{ Profesor titular del Área de Métodos de Investigación y Diagnóstico en } \\
\hline \multicolumn{2}{|l|}{ Educación de la Universidad Autónoma de Madrid. Coordinador del } \\
\hline \multicolumn{2}{|l|}{ Doctorado en Educación de la UAM. Coordinador del grupo de } \\
\hline \multicolumn{2}{|l|}{ Investigación Cambio Educativo para la Justicia Social (GICE). Fue } \\
\hline \multirow{2}{*}{\multicolumn{2}{|c|}{ Coordinador General del Laboratorio Latinoamericano de Evaluación de }} \\
\hline \multirow{2}{*}{$\begin{array}{l}\text { la Calidad de la Educación (LLECE), de la UNESCO, y Director de } \\
\text { Estudios del Centro de Investigación v Documentación Educativa (CIDE) }\end{array}$} & \\
\hline & ResearchGate \\
\hline \\
\hline \multirow{2}{*}{\multicolumn{2}{|c|}{$\frac{\text { Iberoamericana de Investigación sobre Cambio y Eficacia Escolar }}{\text { (RINACE), Director de REICE. Revista Iberoamericana sobre Calidad, }}$}} \\
\hline & \\
\hline \multicolumn{2}{|l|}{ Eficacia y Cambio en Educación, de la Revista Iberoamericana de } \\
\hline \multicolumn{2}{|l|}{ Evaluación Educativa y de la Revista Internacional de Educación para la } \\
\hline \multicolumn{2}{|l|}{ Justicia Social. Ha trabajado como consultor experto en Investigación y } \\
\hline \multirow{2}{*}{\multicolumn{2}{|c|}{$\begin{array}{l}\text { Evaluación Educativas en diferentes países de América Latina, y con } \\
\text { distintas agencias internacionales -UNESCO, OCDE y Convenio Andrés }\end{array}$}} \\
\hline & \\
\hline \multicolumn{2}{|l|}{ Bello-. Su dirección postal es: Facultad de Formación de Profesorado y } \\
\hline Educación. Universidad Autónoma de Madrid. Avda. Tomás y Valiente, & \\
\hline & \\
\hline
\end{tabular}

Hernández-Castilla, Reyes (reyes.hernandez@uam.es).

Profesora Doctora de Métodos de Investigación y Diagnóstico en Educación, Universidad Autónoma de Madrid. Codirectora de laRevista Internacional de Educación para la Justicia Social (RIEJS). Miembro del grupo de Investigación Cambio Educativo para la Justicia Social (GICE) donde es coordinadora de la línea Docencia para la Justicia Social. Miembro de la Red de Investigación sobre Liderazgo y Mejor Educativa, RILME. Ha sido coordinadora de estudios de evaluación de carácter internacional como el TIMSS y el IAEP. Directora de estudios de la empresa Datagrupal, dedicada a Evaluación e Investigación en Educación. Su dirección postal es: Facultad de Formación de Profesorado y Educación. Universidad Autónoma de Madrid. Avda. Tomás y Valiente, 3. 28049 Madrid.

\section{RELIEVE}

\section{Revista ELectrónica de Investigación y EValuación Educativa E-Journal of Educational Research, Assessment and Evaluation}

[ISSN: 1134-4032]

(C) Copyright, RELIEVE. Reproduction and distribution of this articles it is authorized if the content is no modified and their origin is indicated (RELIEVE Journal, volume, number and electronic address of the document).

(C) Copyright, RELIEVE. Se autoriza la reproducción y distribución de este artículo siempre que no se modifique el contenido y se indique su origen (RELIEVE, volumen, número y dirección electrónica del documento). 